\title{
INCONSISTENT BOUNDARIES
}

\author{
ZACH WEBER AND A. J. COTNOIR
}

\begin{abstract}
Mereotopology is a theory of connected parts. The existence of boundaries, as parts of everyday objects, is basic to any such theory; but in classical mereotopology, there is a problem: if boundaries exist, then either distinct entities cannot be in contact, or else space is not topologically connected [Varzi, 1997]. In this paper we urge that this problem can be met with a paraconsistent mereotopology, and sketch the details of one such approach. The resulting theory focuses attention on the role of empty parts, in delivering a balanced and bounded metaphysics of naive space.
\end{abstract}

\section{Introduction: Boundary Disputes}

There is a Great Red Spot on the planet Jupiter. It is a gigantic anticyclonic vortex, rotating counterclockwise. Although the Spot is a dynamic storm, fluctuating remarkably in size and shape, it is persistent and stark, visible through Earth-based telescopes. ${ }^{1}$ What is it that makes the Great Red Spot a distinctive and easily grasped object in our macrophysical experience?

A basic and compelling answer to this question is: a boundary. It goes back to Euclid, who tells us, "a figure is given by its boundaries" (Book I, Def 14). The boundary is what makes there be a figure there at all, and not merely a disenfranchised mess of unrelated stuff. If there is anything to such a proposal, then boundaries would seem to do explanatory work. The notion of boundary is pervasive in ordinary thought about objects. As Varzi puts it, "whether sharp or blurry, natural or artificial, for every object there appears to be a boundary that marks it off from the rest of the world" [Varzi, 2004]. In terms of understanding or representing the world, boundaries appear to be a crucial aspect of the 'naive space' of experience. ${ }^{2}$

Ubiquitous, ordinary and helpful as they may be, however, boundaries are puzzling. Perhaps the most famous problem concerning boundaries is Peirce's Puzzle [Peirce, 1933]. Consider the Great Red Spot on Jupiter, and trace a path from a red-colored point inside the Spot to a non-red colored point outside the Spot. What happens as we pass through the boundary of the Spot? One might think we simply pass through the last red point and then through the first non-red point. But on the assumption that space is continuous, there would be an infinite number of points in between.

\footnotetext{
${ }^{1}$ See [Rogers, 1995, pp. 191-196].

${ }^{2}$ This project can be taken as an exercise in pure metaphysics, naive physics, or spatial representation (at least). By not committing to whether e.g. the problems raised are epistemic, or ontological, etc., our results may be useful to researchers in any of the above areas. Our aim is to work with a naive view of things like the Great Red Spot as continuous objects in continuous space, treating objects as regions of space, and spaces as objects.
} 
What color are they? Are they colorless? Could there be a single point that is both red and not red? Similarly, to which of the Great Red Spot and the rest of the Jovian atmosphere does the boundary belong? Perhaps it belongs to both. Perhaps it belongs to neither. But then either some point both is and is not part of the Great Red Spot, or else there is a gap, a conceptual and spatial hole on the face of Jupiter. It is arbitrary simply to assign the boundary to the Spot, say, and leave its complement in the atmosphere boundariless [Varzi, 2004], or vice versa; it is incomplete not to assign the boundary at all; but the remaining alternative is inconsistent.

These puzzles are symptomatic of the fact that the that following pre-theoretic notions are jointly inconsistent [Varzi, 1997], [Casati and Varzi, 1999, p 74]:

(i) boundaries exist

(ii) space is topologically connected

(iii) discrete entities can be in contact

The purpose of this paper is make these intuitive conditions formally precise in such a way as to be all co-tenable. Section $\S 2$ makes the problem acute by explaining the relevant notions of connectedness, contact, and symmetry that drive the puzzle. Section $\S 3$ considers the options, arguing that there is prima facie reason for a non-classical 'glutty' approach to be investigated. Section $\S 4$ develops paraconsistent mereology, putting focus on the need for empty parts. Section $\S 5$ then sketches the mathematics of boundaries in paraconsistent mereotopology, showing how these 'empty parts' make connected space with bounded subspaces possible. Finally, section $\S 6$ discusses the metaphysics of these results.

We do not pretend to prove that a glut-based approach is the only workable option for a metaphysics of boundaries. Given the state of play, something more basic suffices: to show that a glut-based approach is tenable. ${ }^{3}$

\section{Symmetry and Connection in Naive Space}

Once we begin thinking seriously about bounded objects, we find two basic, interrelated constraints: a strong intuition about symmetry, stemming from the principle of sufficient reason; and a conviction that space is continuous - or topologically connected. These two constraints seem inalienable. They are also incompatible. Thus we have the makings of a precise and perplexing dilemma, of the sort amenable to treatment by non-classical logic.

The purpose of this section is to motivate a formal development of paraconsistent mereotopology. No formal theory - classical, paraconsistent, or otherwise - is presumed; we are comparing

\footnotetext{
${ }^{3}$ This is to set aside other views and issues involving vagueness, the 'problem of the many', supersubstantivalism, and other dialectical alternatives (e.g. about what it means to be in contact, whether topological properties can change over time, whether or not there is a privileged class of 'unified' objects as in [Koslicki, 2008]) — required, if we are to have room to give a formalization of one (reasonably attractive) view that has yet to receive sufficient attention. For recent discussions of similar material, see [Varzi, 1997], [Casati and Varzi, 1999, p 74], [Dainton, 2010, ch. 17], [Hudson, 2005, chs. 2, 3], [Priest, 2006, chs. 11, 12, 15], and [Arntzenius, 2012, ch. 4].
} 
some pre-theoretic notions about space and objects with their standard formalization, using mathematical/philosophical English. The pre-theoretic notions are meant to be graspable enough to understand the problem, and to see the mis-match with orthodox treatment, thus calling out for (but not presupposing) a rigorous treatment.

2.1. Symmetry. Gödel once remarked that, if a geometric line segment is divided evenly at a point, it would be natural and intuitive to expect the two halves of the line to be perfectly symmetric mirror images. ${ }^{4}$ As the continuum is classically constructed, this is not so. The 'center' point must fall either to the left or to the right, leaving one side open and the other side closed; these are the basic notions of topology, wherein a closed object contains its boundary, and an open object does not. (Lines that can be symmetrically divided, such as the rational line around $\sqrt{ } 2$, are not continuous.) And what goes in one dimension, also goes in higher dimensions: the Great Red Spot of Jupiter is, on the received model of space, a set of points that must be open or closed. A boundary seems to split a (connected) space into two parts-but, crucially, one part will be open and the other closed (see $\S 2.2$ below).

The problem now is simple: given no other facts about the space, why do the open/closed properties fall where they do? Neither macroscopic analysis nor mathematical argument would seem to settle the matter, since physically the object loses its definition at very small scales, and logically, the properties of being open and closed may be interchanged without any rational difference [Pratt-Harmon, 2007, p. 14]. We feel the force of the following constraint:

Symmetry: If there is no principled difference between two objects, then there is no principled difference regarding their boundaries, either.

To clarify what Symmetry is not claiming, consider the division of the surface of Jupiter into to halves, one the fusion of points strictly above the equator, and the other the fusion of the equator with the points below the equator. The asymmetry between these two halves in this instance is not at all surprising. ${ }^{5}$ Rather, what is cries for an explanation of some sort is that there is no way to divide the surface in two without one being open and the other closed wherever they meet; the Symmetry intuition is that there should be some way to divide an object without requiring one half to be different from the other. ${ }^{6}$ Symmetry is violated when every way of dividing objects forces an arbitrary difference.

To put Symmetry the other way around, then, if there is a difference between the boundaries of two objects, a principled and explicable difference that persists across all cases, then there is an

\footnotetext{
${ }^{4}$ According to Putnam, citing a short unpublished note [Putnam, 1994].

${ }^{5}$ Thanks to referees for pressing this point.

${ }^{6}$ If neither an object nor its complement are fully bounded, both are partly open and partly closed. As such, the object has a proper part that is closed, and therefore the complement's bordering part must be open, and vice versa. This defers the problem to a smaller scale; see [Casati and Varzi, 1999, p.80].
} 
explicable difference between the objects after all. ${ }^{7}$ Where one object is open and another is closed, there should be some reason as to why; but to ask which is which about Jupiter and its Spot is to ask is "an embarrassing question" [Casati and Varzi, p 87]. Without some further mitigating reason, it is hard to take as a brute fact that some objects include their boundary as a part while others do not. ${ }^{8}$ The Symmetry problem is the postulation of a distinction without a difference.

2.2. Connectivity. The bite of Peirce's Puzzle comes from the assumption that space is connected. The key fact is just this:

Separation: A space is separated iff there is an exclusive and exhaustive division of the space into two closed parts.

(Cf. [Hocking and Young, 1961, p.14].) Spaces that are not separable are connected. (As per footnote 2, all this goes equally for objects.) Since the basics of topology tell us that the complement of a closed set is open [Kelley, 1955, p. 40], both parts of the partition in a separation are open, too. Connectivity is enmeshed with the concepts of open and closed sets.

Let us draw the conventionally-understood interplay of these concepts a bit more sharply, by imagine the following scenario. There is about to be peace in the middle east. The leaders of three countries have struck an agreeable deal, and are about to shake hands. First $A$ shakes with $B$, so their hands are in contact; therefore (without loss of generality) $A$ 's hand is topologically open, and $B$ 's hand is topologically closed. Then $B$ shakes with $C$; so (again, without loss of generality) $B$ is closed and $C$ is open. Finally, $A$ shakes with $C$. Either $C$ 's hand changes from open to closed, or else both have open hands, and therefore cannot come into contact. That is, no matter how hard they push, they cannot truly shake hands! As far as naive spatial representation goes, this is nonsense.

What this shows is that a space that satisfies the SYMMETRY intuition cannot, apparently, be connected. ${ }^{9}$ And this is itself a problem, because the space of naive experience is connected. There are no rips or tears. But here is the important part. In classical topology, that is, topology embedded in classical logic, SEPARATion can be rephrased: a topological space is connected (not separable) if and only if the only the only sets that are both open and closed are the entirety of space, and the empty set [Kelley, 1955, p. 53], [Hocking and Young, 1961, p.14, lemma 1-11].

Why? How is this rather recondite expression of connectivity a re-statement of intuitive claims about space? Well, consider an apparently connected space like Jupiter, and some non-empty proper subset of it, like the Great Red Spot, $G$. Suppose $G$ is both open and closed. The boundary of $G$,

\footnotetext{
${ }^{7}$ Interestingly, this is itself a continuity intuition - roughly, if $a$ is very similar to $b$, then $\partial(a)$ is very similar to $\partial(b)$, making the boundary operator like a continuous function.

${ }^{8}$ See [Dummett, 2000, p. 505] and [Zimmerman, 1996, p. 12] for more problematizing the metaphysics, and [Sider, 2000] for a possible reply.

${ }^{9}$ Casati and Varzi try to relocate the asymmetry [Casati and Varzi, 1999, p.80, 87], but the fundamental forced choice remains: as ever, in cutting a continuous line in half, there is a single point that either falls on the left or on the right, but not both-an asymmetry.
} 
intuitively, is made up of all the points that are 'extremely close' to both $G$ and its complement $\bar{G}$. Write $\partial(G)$ for the boundary of $G$. (A precise definition of $\partial$ is given in $\S 5$.) Now, if $G$ includes its boundary, if it is closed, then this means $\partial(G)$ is a subset of $G$. If $G$ is open, then $\partial(G)$ is a subset of $\bar{G}$. Ergo if $G$ is both open and closed, then for any $x \in \partial(G)$,

$$
x \in G \quad \text { and } \quad x \notin G
$$

which is a contradiction. By classical reductio, then, the boundary is empty, $\partial(G)=\varnothing$. Therefore if the Great Red Spot is really a non-empty proper subspace of Jupiter's surface, then there is a rip in the fabric of the space, and so the space is not connected. The existence of bounded, discrete entities seems to grate against the connectivity of space (cf. $\S 5.2$ ).

Our reasoning seems to have led us into a corner. On the one hand, objects that are touching share a boundary; space is connected. On the other hand, objects do not arbitrarily differ. But given our two objects, if the boundary belongs to one, then that object is closed, and ipso facto the other, by virtue of connection. If a sub-object in space is both open and closed, then the space is not connected at all.

The task ahead is to disentangle this thicket of intuitions, so that proper, non-empty subspaces of a connected space may be both open and closed.

\section{Options}

Aristotle famously defined the boundary of an object as "the first thing outside of which no part is to be found, and the first thing inside of which every part is to be found" (Metaphysics 1022a), and the germ of his definition is retained in modern topology. But we have seen that modern topology may not settle the matter. Assuming the connectivity of space, there are some theoretical options:

(1) Classical: A boundary may be part of an object or its complement, but not both.

(2) Glutty: A boundary may be part of both an object and its complement.

(3) Gappy: A boundary may be neither part of an object nor part of its complement.

(4) Coincidence: There are two coinciding boundaries: one that is part of an object, and one that is part of its complement.

(5) Eliminativist: An object really has no boundary at all; boundaries are either abstractions (equivalence classes) of convergent series of nested bodies, or the boundary of the space-time receptacle of that object, etc. 
Philosophers have defended each of these views. ${ }^{10}$ Only three of these theories have been made mathematically precise. The Classical view - which is so called due to its basis in 'classical' mereotopology — has been historically dominant, and developed formally in [Casati and Varzi, 1999]. Following Chisholm, the CoIncidence view is formalized in [Smith, 1997]. At least two versions of Eliminativism have been given precise formalizations. The Whiteheadian theory is fully formalized in [Clarke, 1985], and extended in [Randell et al., 1992]. ${ }^{11}$ However, to our knowledge there have been no GAPPY or GLUTTY formal theories put forward. ${ }^{12}$

As we have been arguing, the CLASSICAL view fails to satisfy the SYMMETRY intuition that a boundary never arbitrarily belong to an object over its complement. The other options all appear to satisfy this constraint. We think the GLUTTY view has the best chance of modeling our naive intuitions about space, objects, and their boundaries. To motivate that, we now point out certain costs of adopting (3)-(5) - not to raise these challenges as if they were insuperable, but to give evidence that the GLUTTY view has some prima facie advantages over its rivals.

3.1. Coincident Boundaries. Recall that the CoInCIDENCE approach claims that any two connected objects have two distinct but coincident boundaries separating them. Indeed, the theory claims that all entities whatsoever have boundaries as parts as a matter of metaphysical necessity. ${ }^{13}$ That is, all entities are closed. In this way, there is no arbitrary choice between two adjacent objects as to which is to be open and which closed. Both must be closed, each containing their own boundary.

It is worth noting that the COINCIDENCE approach requires a great deal more coincident objects than one might expect.

Each point within the interior of a two- or three-dimensional continuum is in fact an infinite (and as it were maximally compressed) collection of distinct but coincident points: punctiform boundaries of straight and crooked lines, of two-dimensional segments of surfaces and of interior regular and irregular cone-shaped portions within three-dimensional continua, etc. (Not for nothing were the scholastic philosophers

\footnotetext{
${ }^{10}$ The standard account of boundary in point-set topology reflects the CLASSICAL view, defended in [Bolzano, 1950]. The Glutty view seems to be found first in [Peirce, 1933], and then [Priest, 2006]. [Varzi, 1997] cites Leonardo as holding a GAPPY view, with additional possible adherents being [Hestevold, 1986] and [Sorensen, 1986]. The Coincidence view is in [Brentano, 1988] and [Chisholm, 1984]. Finally, the Eliminativist is most notably in [Whitehead, 1919]. NB that the use of the term CLASSICAL does not indicate that all other approaches are based in a non-classical logic.

${ }^{11}$ To be accurate, there are a number of different boundary-free theories. Lumping all such theories under the category of EliminativiSM is simply for ease of explication, and because below we will prefer boundaried theories over boundariless ones.

${ }^{12}$ The closest thing anyone has put forward is the bi-Heyting algebraic theory of Stell and Worboys [Stell and Worboys, 1997]. This algebra is developed primarily to yield point-free models of the Region Connection calculus of [Randell et al., 1992]. This structure is importantly different from the one we propose below. In particular, it is based in classical logic. But a full examination of those differences will have to wait for another time. ${ }^{13}$ See [Smith, 1997, p. 18].
} 
exercised by the question as to how many zero-dimensional beings might be fitted onto the head of a pin.) [Smith, 1997, p. 10]

Again, consider the real line $\mathbb{R}$. Consider the boundary between the intervals $[-1,0]$ and $[0,1]$, and the boundary between the intervals $[-2,0]$ and $[0,2]$, and the boundary between $[-3,0]$ and $[0,3]$, and so on. Even simply considering only the natural number intervals, there are infinitely many coincident boundaries stacked up at 0 . For those with concerns of ontological parsimony, the view appears ontologically inelegant, other things being equal. Of course, ontologically bloated theories are sometimes much more elegant than parsimonious ones, when other things aren't equal; and those unconcerned with parsimony considerations of this kind will not find the view inelegant.

Of more pressing importance is that the COINCIDENCE tells us that there are many otherwise indistinguishable boundaries, all co-located and doing the same work. So this is going astray in the same way that violations of SYMMETRY do, namely by postulating a distinction without a difference. Indeed, there is a sense in which CoINCIDENCE theory does not, in fact, satisfy the symmetry constraint. While there is no arbitrary choice between bounded and boundariless objects, there still is a choice as to which object a given boundary belongs. The Great Red Spot has a boundary $b_{1}$ and so does its complement $b_{2}$. But given their coincidence, what makes it the case that it is $b_{1}$ and not $b_{2}$ that is the Spot's boundary? Is there any non-arbitrary answer? A given boundary is always directed toward the (only) entity that it bounds; boundaries are essentially directional without any metaphysical explanation for their direction. Of course, boundaries may have only one direction (e.g. the intersecting point of a circle and a tangential line is a boundary in only one direction) or many directions (e.g. a point in the interior of a solid sphere is a boundary in every direction). But the problem remains that, according to CoINCIDENCE, boundaries are attached to their objects in an essentially asymmetrical way.

3.2. Eliminativism about Boundaries. The Eliminativist views also do not commit the error of assigning boundaries arbitrarily, simply because they do not assign boundaries at all. It may seem that this way of accommodating SYMMETRY results in too few boundaries.

First, on such a view, all objects are unbounded and thus, it is unclear how the open/closed distinction can be recovered in full generality. Now, perhaps some boundariless systems are able to recover some topological notions, and here is not the place to launch a full investigation into the issue. It is worth noting, however, that the above problem regarding the impossibility of handshakes at peace talks arises again with a vengeance. Assuming the connectedness of space, no two objects can touch as neither includes their boundary. The call for explanation rings as loudly as ever.

Secondly, the openness of all objects gives rise to a new puzzle. Imagine a completely homogeneous sphere (in the Eliminativist case, imagine it is open); but consider it as already divided into two halves. Since there are no boundaries, neither half is bounded on its inside. It seems implausible to suppose it impossible to fuse these halves back together into their original spherical 
shape. But if it is possible, then fusing the two halves together will have to be a process of creating the 'missing' boundary points without which there would be a gap in the center of the sphere. To put the point differently, consider the open intervals $(-1,0)$ and $(0,1)$ in $\mathbb{R}$. Note that the point 0 cannot exist, otherwise the boundary of the intervals would exist, contra eliminativism. What is the mereological sum of these two objects? Presumably, the intended sum is the interval $(-1,1)$. But that includes 0 which was supposed not to exist. And if a sum fails to include 0 , why the gap in the center?

Eliminativism may yet be tenable, but something must be said to explain the newfound awkwardness. As it stands, the proposed solution fails to solve the original SYMMETRY puzzle.

3.3. Gappy Boundaries. Finally, we consider the GAPPy option. As this option is closest to our preferred theory, we won't have much to say against it. There are, however, some puzzles that gappiness raises that are not faced by the glutty approach.

What separates Great Red Spot from its complement? Well, since we're not being eliminativists, a boundary exists between them. If a 'gap', the boundary is neither part of the Spot nor its complement. But now we seem to have a new question: since the boundary and the Spot are disjoint, what separates the boundary from the Spot itself (alternatively, what separates the boundary from the Spot's complement)? Presumably, by parity of reasoning, there must be a new boundary between the Spot and its boundary. And if a 'gap', this boundary is part of neither the Spot, nor the boundary of the Spot. So what separates the Spot from this new boundary? As is obvious, we have found ourselves in a regress such that there are infinitely many distinct gaps between any two objects in contact. ${ }^{14}$ Now, whether this regress is vicious is an interesting question. But it is an open question nonetheless. A related problem arises with the gappy view in light of the classical truth that the boundary of the boundary of an object is part of that object. But the GAPPY view seems to imply that the boundary of a boundary cannot be part of the object.

All other options considered, we propose that it is time for a serious investigation of a GLUTTY account, according to which the boundary between two objects in contact is part of both.

\section{Mereology: Parts, Overlap, and Identity}

The formally precise treatment of concepts surrounding parts in connected space is called mereotopology, built by adding standard topological operators to mereology. Work in this formal discipline until now has been conducted under the auspices of classical logic. In this section, we will develop a paraconsistent mereology which forms the basis for a metaphysics of glutty boundaries. ${ }^{15}$

\footnotetext{
${ }^{14}$ Thanks to Marcus Rossberg for raising this point, which is reminiscent of the problem of higher-order vagueness. ${ }^{15}$ There is a school of thought, owing to Poincaré, Weyl, and Whitehead, that the problems associated with boundaries are in fact problems about conceptualizing space in terms of points. This is an idea is preserved in point-free (or 'pointless') geometry; see the recent [Arntzenius, 2012, ch. 4]. Some in fact take mereotopology to be a recasting of geometry in terms of qualitative regions, rather than quantitative points (or sets of points) [Pratt-Harmon, 2007], and so the move to mereology itself to be a solution to the puzzles.
} 
4.1. Basic Mereology. Here is a brief introduction to classical mereology, using a slightly nonstandard axiomatization from [Hovda, 2009, Part 4]. This axiomatization is equivalent to those in more standard presentations such as the axiomatization of 'general extensional mereology' (GEM) in [Casati and Varzi, 1999], or that of 'classical extensional mereology' (CEM) in [Simons, 1987]. The axiomatization highlights some features which will be important when we move to a GLUTTY approach.

Let HM0 be an axiomatization of classical logic with identity $=$, and with $\supset$ the material conditional. Then HM0-HM6 below is an axiomatization of classical mereology. Parthood, $\leqslant$, is taken to be primitive and is a partial order.

HM1: $\forall x \quad x \leqslant x$

HM2: $\forall x \forall y((x \leqslant y \wedge y \leqslant x) \supset x=y)$

HM3: $\forall x \forall y \forall z((x \leqslant y \wedge y \leqslant z) \supset x \leqslant z)$

From parthood, we can define notions like overlap, disjoint, and proper part.

$$
\begin{aligned}
& x \bullet y \quad=_{\mathrm{df}} \quad \exists z(z \leqslant x \wedge z \leqslant y) \\
& x \mathfrak{l} y==_{\mathrm{df}} \quad \neg x \bullet y \\
& x<y \quad=_{\mathrm{df}} \quad x \leqslant y \wedge \exists z(z \bullet y \wedge z \mathbb{1} x)
\end{aligned}
$$

Two objects overlap when they have a common part, and two objects are disjoint when they have no parts in common. Proper parthood is the corresponding strict order to parthood; the definition here is slightly unorthodox, but it turns out to be better to work with in a paraconsistent setting, since it makes the difference between $x$ and $y$ explicit. Given extensionality, this definition is classically equivalent to the more usual ones like $x \leqslant y \wedge y \$ x$.

A basic axiom guarantees the existence of complements:

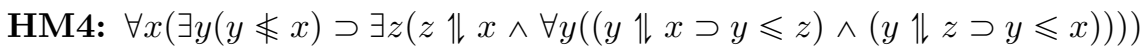

The axiom states that if an object $y$ isn't part of another $x$, then there is an object, $\bar{x}$, made up of all and only the non-x-overlapping parts of $y$. The antecedent allows there not to be a complement to the whole universe, which would be the 'empty object' were it to exist (as such, this axiom is consistent with HM6, below). The axiom asserts that objects do not overlap their own complements. One can prove that $y \leqslant \bar{x} \supset y \mathfrak{l} x$ by reductio. ${ }^{16}$ Moreover, this axiom entails the standard equivalence of $x \leqslant y$ with $\forall z(z \bullet x \rightarrow z \bullet y) .{ }^{17}$

\footnotetext{
${ }^{16}$ Strictly speaking, HM1 is redundant, since the Boolean complement axiom HM4 implies the reflexivity of parthood. Proof: If $\forall x(\exists y y \leqslant x)$ is false, then $\exists x \forall y y \leqslant x$. Hence, $x$ is the universe, and $x \leqslant x$. If $\forall x(\exists y y \leqslant x)$, then find the complement $\bar{x}$. As above, $x$ 1 $\bar{x}$. By HM4, $\forall y(y$ \ $\bar{x} \supset y \leqslant x)$, so $x \leqslant x$.

${ }^{17}$ Cf. Remark 4 below. Standard axiomatizations of mereology usually include a supplementation axiom-the usual candidate is Strong Supplementation: $\forall x \forall y(y \$ x \supset \exists z(z \leqslant y \wedge z 1 x))$. This stipulates a 'remainder' in cases where one object is not part of another. One instance of $y \leqslant x$ is the case where $x<y$. In that case, supplementation is used to guarantee that if $y$ has a proper part, then it must have another. Contraposing gives the crucial direction of this equivalence. See [Hovda, 2009, Part 4] for how our axiom set is equivalent to ones with e.g. supplementation.
} 
Fusions of definable collections of objects exist; such objects combine to make a whole. Here, fusions are least upper bounds:

$$
\operatorname{lub}(t, A)=_{\mathrm{df}} \forall x(A \supset x \leqslant t) \wedge(\forall w \forall x(A \supset x \leqslant w) \supset t \leqslant w)
$$

So a lub of the $A$ s is just an object that has all the As as parts (i.e. an upper bound), and is part of any other upper bound of the As (i.e. least). Classical mereology stipulates that fusions always exists for any (definable) collection of $A \mathrm{~s}$ :

HM5: $\exists x A \supset \exists z \operatorname{lub}(z, A)$

A classical axiomatization, finally, ensures there is no empty object; that is no object which is a part of everything. This is a standard part of classical mereology, and it is usually derived from supplementation principles. ${ }^{18}$

HM6: $\exists x \exists y(y \neq x) \supset \neg \exists x \forall y(x \leqslant y)$

HM6 states that, so long as there are at least two things, there is nothing which is a part of everything.

Empty objects have almost always been excluded from mereology, on the grounds that an 'empty fusion' is highly counterintuitive, although there are some who have argued in its favor (e.g. [Carnap, 1947], [Bunge, 1966], [Bunt, 1985], [Martin, 1965], and in a paraconsistent context, [Priest, 2014]; cf. [Oliver and Smiley, 2013]). Calling it a 'fiction', Simons calls advocates of it "culprit[s] in propounding this absurdity", and is relieved to find "the idea is neatly dispatched in Geach 1949 and we need not take it seriously" [Simons, 1987, p. 13]. The matter is not so neatly dispatched, however. Geach devotes only a few lines to this thought [Geach, 1949, p. 522], exclaiming that "the null thing is described as ... existing nowhen and nowhere!" Incredulity is not an argument. In what follows, empty parts will be play a distinctive role in a GLUTTY approach.

Axioms HM1-HM6 makes plain the fact that models of classical mereology are complete Boolean algebras without a 'zero' element.

4.2. Enter Paraconsistency. We have argued that there is a sufficiently intuitive conception of boundaries to be formalized. One aspect of that conception is expressed by the SYMMETRY constraint, as illustrated by the simple universe in Figure 1 . Call the whole universe $\mathbb{1}$; we also have an object $a$, an object $\bar{a}$ which is $a$ 's complement in $\mathbb{1}$, and the intuitive, non-empty boundary between them, $b$. (All these notions will be made precise in $\S 5$.) Where does $b$ belong? Is $b \leqslant \bar{a}$ or is $b \leqslant a$ ? According to classical mereology cannot be part of both. If $b$ were part of both then $a$ would overlap its complement. But such overlap violates HM4, and is classically impossible.

Our competing intuitions are irreconcilable in a theory with classical logic in the background. What of a theory with a paraconsistent logic in the background? Paraconsistent logics reject ex

\footnotetext{
${ }^{18}$ Assume for reductio that there are at least two objects 0 and 1 , and $\forall x \leqslant x$. Note, $\mathbb{1} \$$ : if it were, $\mathbb{1} \leqslant \wedge \leqslant \mathbb{1}$ which by HM2 implies $=\mathbb{1}$. But that is contrary to our supposition. So by e.g. Strong Supplementation, $\exists z(z \leqslant \mathbb{1} \wedge z 1 /)$. But nothing is disjoint from, since it is part of everything. Contradiction.
} 


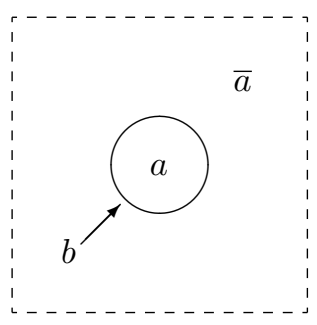

Figure 1. A Simple Universe

falso quodlibet, the inference $A \wedge \neg A \vdash B$, by weakening the classical Boolean negation to a De Morgan negation. This negation satisfies double-negation elimination, the De Morgan laws, and excluded middle; it does, however, allow for some types of inconsistency. The crucial property, which will emerge in constructions as we go, is the invalidity of disjunctive syllogism:

$$
A \vee B, \neg A \nvdash B
$$

(Let $A$ and $\neg A$ both hold; then $B$ could be absurd.) The logic is found in $\S$ Appendix 1 .

By moving to a paraconsistent logic with its corresponding De Morgan negation, we can have a diagram of our Simple Universe (Figure 2) that satisfies SYMMETRY. The nodes are objects, and paths via upward arrows indicate proper parthood. ${ }^{19}$

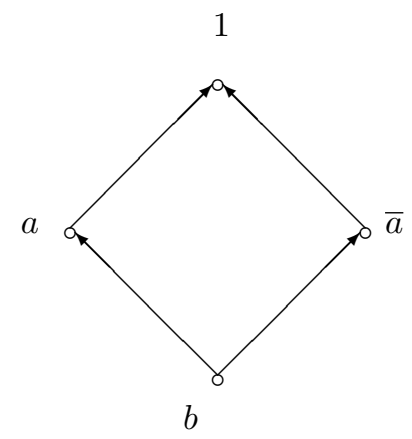

Figure 2. Paraconsistent Model of a Simple Universe

We can allow that $b \leqslant a$ and $b \leqslant \bar{a}$, even in the presence of complementation axioms. This is because now being disjoint, $a \mathbb{k} \bar{a}$, may hold simultaneously with overlap, $a \bullet \bar{a}$. Indeed, in general complementation does not rule out disjoint objects $a$ and $\bar{a}$ sharing parts. What follows is $a \bullet \bar{a} \wedge \neg a \bullet \bar{a}$. The boundary of $a$ is inconsistent; the boundary is also symmetric.

\footnotetext{
${ }^{19}$ This diagram and others below are intended to be illustrative only - i.e. not presented as a consistency argument for the theory. For such an argument, see $\S$ Appendix 2.
} 
In classical theory, HM6 rules out an empty object because nothing would be disjoint from it - an empty object is part of everything. In the proposed paraconsistent recasting, Figure 2 has an obvious bottom element, $b$. But it is important to note that this sort of 'emptiness' is distinct from the 'empty object'. In a paraconsistent context, an object's parts (the $x$ s for which $x \leqslant y$ ) are independent of its non-parts (the $x$ s for which $x \$ y$ ). That is, it is paraconsistently acceptable that an object's part might also be a non-part. This is precisely the case with $b$ and $a$ above: $b \leqslant a$ while $b \$ a$. Now, a classically empty object would be such that $\leqslant x$ for all $x$ and $\$ x$ for no $x$. In the case above, $b$ isn't classically empty, since it is among $a$ 's non-parts. Another way of putting the point is that $b$ satisfies the axiom HM6, which rules out the 'empty object'. ${ }^{20}$ As we will see again, $b$ is not truly 'empty' even though it is part of everything else.

That the algebra for a paraconsistent mereology naturally points to the existence of such 'empty' elements, turns out to be the skeleton key unlocking the whole metaphysical riddle. (See $\S 6$.)

Let PM0 be an axiomatization for a paraconsistent logic (given in the $\S$ Appendix 1). Then:

PM1: $\forall x x \leqslant x$

PM2: $\forall x \forall y((x \leqslant y \wedge y \leqslant x) \leftrightarrow x=y)$

PM3: $\forall x \forall y \forall z((x \leqslant y \wedge y \leqslant z) \rightarrow x \leqslant z)$

PM4: $\forall x(\exists y(y \leqslant x) \mapsto \exists z(z \mathbb{\|} x \wedge \forall y((y \mathbb{\|} x \rightarrow y \leqslant z) \wedge(y \mathbb{\|} z \rightarrow y \leqslant x))))$

PM5: $\exists x A \mapsto \exists z \operatorname{lub}(z, A)$

PM6: $\exists x \exists y(y \neq x) \mapsto \neg \exists x \forall y(x \leqslant y)$

PM7: $x \leqslant y \mapsto \forall z(z \bullet x \rightarrow z \bullet y)$

where $\bullet$ and $1 \mathfrak{l}$ are defined as above, and

$$
\operatorname{lub}(t, A)=_{\mathrm{df}} \forall x(A \mapsto x \leqslant t) \wedge(\forall w \forall x(A \mapsto x \leqslant w) \mapsto t \leqslant w)
$$

PM0-PM7 axiomatizes our paraconsistent mereology. Again, PM1-PM3 give a partial order. PM5 generates a lub for every (definable) subset. PM6 rules out the purely empty object, and likewise forces any bottom element of our structures to be among the non-parts of some other object. With a non-classical negation, PM4 gives De Morgan complements, $\bar{x}$ for every $x$ (excluding the universe). So these structures correspond to complete De Morgan Algebras without 0. PM7 expresses an important relationship between parthood and overlap; it is a theorem of classical mereology, but fails to be provable from PM1-PM6 due to the relative weakness of the paraconsistent background logic.

Note on metatheory. The theory PM to be developed is non-trivial (or 'absolutely consistent'), in the sense that it has a classical model in which some sentence is not satisfied. This is shown, via a classical metatheoretic argument, in $§$ Appendix 2. In the next sections, when we describe things over paraconsistent mereology by giving models, we are availing ourselves of that same classical

${ }^{20}$ Why? Well, since we have more than one object around, HM6 gives $\neg \exists x \forall y x \leqslant y$, which is just equivalent to $\forall x \exists y x \leqslant y$. But $a$ is a witness for $y$ when $x$ is instantiated by $b$. 
metatheory, and flag this by labeling them 'FACT'. When we give axiomatic proofs, these are taken to be entirely within the theory, and are numbered theorems.

4.3. Basics: Parthood, Overlap, and Extensionality. Mereological objects have several important properties. A fundamental relationship is

$$
x \leqslant y \mapsto x \bullet y
$$

For $x \leqslant y \wedge x \leqslant x \rightarrow \exists z(z \leqslant y \wedge z \leqslant x)$. Since $x \leqslant x$ always holds by PM1, the enthymematic $\mapsto$ conditional lets us suppress the assumption. The contrapositive does not hold: the proof shows only that $\neg x \bullet y \rightarrow x \nless y \vee x \$ x$. Classically, PM1 would eliminate the $x \$ x$ disjunct, by disjunctive syllogism - but not so here. Similarly, if $x \leqslant y$ and $z \bullet x$, then $z \bullet y$, by transitivity PM3. But this argument is a long way from establishing anything with the stronger paraconsistent conditional $\rightarrow$.

To give more of a feel for the system, then, let us look at other classically expected properties that do not hold in the paraconsistent setting. In classical mereology, we have the extensionality of proper parthood: any composite objects - objects $x$ such that $\exists y(y<x)$ - that have the same proper parts are identical. Not so here:

FACT 1 Some composite objects have the same proper parts, but are not identical.

Consider again the Simple Universe as depicted in Figure 1, and its model in Figure 2. Notice that $a$ and $\bar{a}$ are both composed objects - that is, they have proper parts. But they have the same proper parts: $b<a$ while $b<\bar{a}$, and neither $a$ nor $\bar{a}$ have any other proper parts. But clearly, $a \neq \bar{a}$. So we have a failure of the extensionality of proper parthood. So, we know generally that having the same parts is not sufficient for identity; things additionally need to have the same non-parts as well. In the above example, $a$ 's non-parts include only $b$ and $\mathbb{1}$ whereas $b$ 's non-parts include only $a$ and $\mathbb{1}$. Hence, they are distinct.

For a second divergence from classical mereology, consider extensionality of overlap:

FACT 2 Some objects overlap all the same things, but are not identical.

Take an extremely simple two-element model, with a point-sized boundary $a$ and the universe $\mathbb{1}$ - which can be thought of the space with $a$ 'removed'. Intuitively, $a$ is its own boundary; as such it is an inconsistent object- that is, $a \leqslant a$ but also $a \leqslant \bar{a}$, and so $a \bullet \bar{a}$. The paraconsistent model for this scenario is depicted in Figure 4.

This model verifies Fact 2. Since $a \bullet \mathbb{1}$ and $\mathbb{1} \bullet \mathbb{1}$ while $a \bullet a$ and $a \bullet \mathbb{1}$, both $a$ and $\mathbb{1}$ overlap exactly the same things. But $a \neq \mathbb{1}$, in particular because they do not have the same non-parts, $\mathbb{1} \$ a$ but $a \leqslant \mathbb{1}$. Moreover, despite overlapping all the same things, $a$ and $\mathbb{1}$ differ with in what they are disjoint from: $a \mathfrak{1} a$ whereas $\mathbb{1}$ is disjoint from nothing. Thus, we have a failure of the extensionality of overlap. 


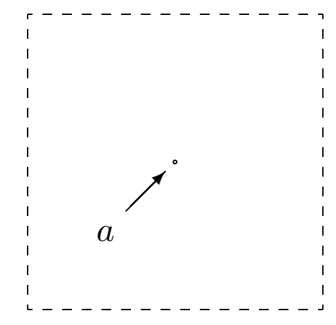

FiguRE 3. A Simpler Universe

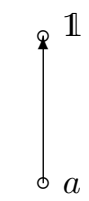

Figure 4. Paraconsistent Model of the Simpler Universe

The model also violates the classically-minded notion of supplementation: that whenever an object has a proper part, it must have another disjoint from the first.But $a<\mathbb{1}$ with no other proper parts. This may seem strange when thinking of parthood classically; but on the paraconsistent picture it makes sense - since $a$ is part of $\mathbb{1}$ but it is also a non-part of $\mathbb{1}$. There need be no remainder of $\mathbb{1}$ without $a$, since $a$ is already 'removed' so to speak. Since boundaries are inconsistent objects, they need not require any further parts of that object to exist (though see Remark 4 below).

A third property is the uniqueness of fusion. This is the first proper theorem of PM; from here on, we will be working entirely inside the paraconsistent mereology, and move to numbered theorems to signal that they are 'official' parts of the (object) theory. ${ }^{21}$

Theorem 1. $\forall u \forall v((\operatorname{lub}(u, A) \wedge \operatorname{lub}(v, A)) \rightarrow u=v)$

This holds in paraconsistent mereology. Let $\operatorname{lub}(t, A)$ and $\operatorname{lub}\left(t^{\prime}, A\right)$. By the definition of fusion, we have $t \leqslant t^{\prime}$ and $t^{\prime} \leqslant t$, which by PM2 gives $t=t^{\prime}$.

4.4. Sums, Products, and Complements. Fusions are guaranteed to exist (by PM5) and are unique (by Theorem 1); so they license definite descriptions: we write lub $[z: A]$ for the least upper

\footnotetext{
${ }^{21}$ Proving a proposition inside a paraconsistent theory means, as elsewhere, that it is true in that theory. (In the model of Appendix 2, it means for the proposition to have value ' $t$ ' or ' $b$ '.) This is paraconsistent logic: a theorem may be taken to be proved, without a guarantee that its negation fails.
} 
bound of the $z \mathrm{~s} \mathrm{s.t.} A(z)$ holds. The existence of a greatest lower bound,

$$
\operatorname{glb}(t, A)=_{\mathrm{df}} \forall x(A \mapsto t \leqslant x) \wedge(\forall w \forall x(A \mapsto w \leqslant x) \mapsto w \leqslant t)
$$

follows from the PM5, too: provided $x$ and $y$ have a lower bound, which they do if they overlap, the fusion (lub) of all their lower bounds is the $\mathrm{glb}$ of $x$ and $y .{ }^{22}$ By Theorem $1, \mathrm{glb}[z: A]$ is unique, too.

This in hand, the lattice properties come to the fore. The binary sum of $x$ and $y$ is

$$
x \sqcup y={ }_{\mathrm{df}} \operatorname{lub}[z: z=x \vee z=y]
$$

and a (binary) product operation $\sqcap$, meaning greatest lower bound — the maximal common part—is

$$
x \sqcap y={ }_{\mathrm{df}} \mathrm{glb}[z: z=x \vee z=y]
$$

provided that $x \bullet y .{ }^{23}$ These have standard behaviors.

Proposition 2. Lattice properties hold:

(i) $x \leqslant y$ iff $x \sqcap y=x$ iff $x \sqcup y=y$

(ii) $x=x \sqcup x ; x \leqslant x \sqcup y$

(iii) $x \sqcup y=y \sqcup x$

(iv) $x \sqcup(y \sqcup z)=(x \sqcup y) \sqcup z$

(v) $x \sqcup(y \sqcap z)=(x \sqcup y) \sqcap(x \sqcup z)$

These follow from the algebra of the underlying logic, e.g. for (iii),

$$
x \sqcup y=\operatorname{lub}[z: z=x \vee z=y]=\operatorname{lub}[z: z=y \vee z=x]=y \sqcup x
$$

Further, from (ii), if $x \leqslant y$ then by transitivity (PM3), $x \leqslant y \sqcup z$.

Complements are guaranteed to exist by PM4, abbreviated thus:

$$
\bar{x} \quad=_{\mathrm{df}} \operatorname{lub}[z: z 1 \mid x]
$$

Then it is assumed in PM4 that nothing overlaps with its own complement-although this last, as we will see, needs to be understood as a paraconsistently violable claim when it comes to boundaries

\footnotetext{
${ }^{22}$ Specifically, $t=\operatorname{lub}[x: w=a \vee w=a \mapsto x \leqslant w]$ iff $\forall x((\forall z(z=a \vee z=b \mapsto x \leqslant z) \mapsto x \leqslant t) \wedge \forall w \forall x(\forall z((z=a \vee z=b \mapsto x \leqslant z) \mapsto x \leqslant w) \mapsto t \leqslant w)$
}

i.e. if $x$ is a lower bound of $a$ and $b$, then $t$ is an upper bound of $x$, and any upper bound of lower bounds of $a$ and $b$ is above $t$. The second conjunct entails that $t$ is a lower bound of $a$ and $b$, i.e. $\forall x((x=a \vee x=b) \mapsto t \leqslant x)$. Uniform variable relabeling of the first conjunct gives: $\forall w \forall x((x=a \vee x=b \mapsto w \leqslant x) \mapsto w \leqslant t)$. Conjoining (in a different order $): \forall x((x=a \vee x=b) \mapsto t \leqslant x) \wedge \forall w \forall x((x=a \vee x=b \mapsto w \leqslant x) \mapsto w \leqslant t)$ iff $t=\operatorname{glb}[w: w=a \vee w=b]$ by definition of glb.

${ }^{23}$ Cf. [Casati and Varzi, 1999, p. 46]. In some cases, the defining condition may not be met, but we will ignore this complication in what follows. 
in connected space. ${ }^{24}$ The product $x \sqcap \bar{x}$ exists only to the extent that some $u \leqslant x \wedge u \leqslant \bar{x}$. (That there is at least one such $x$ is explicitly assumed in the logic; see $\S$ Appendix 1).

Complementation is extensional enough, from PM7, to help prove standard theorems:

Proposition 3. The binary operations are De Morgan:

(i) $x \leqslant y \mapsto \bar{y} \leqslant \bar{x}$

(ii) $x=\overline{\bar{x}}$

(iii) $\overline{x \sqcup y}=\bar{x} \sqcap \bar{y}$

(iv) $\overline{x \sqcap y}=\bar{x} \sqcup \bar{y}$

(v) For all $u, u \leqslant x \sqcup \bar{x}$ and $u$ 1 $x \sqcap \bar{x}$.

Proof. Compute. For (i), assume $x \leqslant y$. Then $\forall z(z \bullet x \rightarrow z \bullet y)$ by PM7, which is equivalent to $\forall z(\neg z \bullet y \rightarrow \neg z \bullet x)$. Since $\neg \bar{y} \bullet y$ by PM4, it follows that $\neg \bar{y} \bullet x$ by modus ponens. Then $\bar{y} \leqslant \bar{x}$ follows, again by PM4.

The antisymmetry axiom PM2 and complementation axiom PM4 yield (ii):

$$
x \mathfrak{x} \wedge \overline{\bar{x}} \mathfrak{h} \bar{x} \rightarrow x \leqslant \overline{\bar{x}} \wedge \overline{\bar{x}} \leqslant x
$$

For (iii), $x \leqslant x \sqcup y$, so $\overline{x \sqcup y} \leqslant \bar{x}$, by (i). Similarly, $\overline{x \sqcup y} \leqslant \bar{y}$; i.e. $\overline{x \sqcup y}$ is a lower bound of both $\bar{x}$ and $\bar{y}$. To show it is greatest, suppose $a$ is also a lower bound: $a \leqslant \bar{x}$ and $a \leqslant \bar{y}$. Then $x \leqslant \bar{a}$ and $y \leqslant \bar{a}$ by (ii) and (i) again. So $x \sqcup y \leqslant \bar{a}$, and hence $a \leqslant \overline{x \sqcup y}$. So $\overline{x \sqcup y}$ is the greatest such lower bound; i.e $\overline{x \sqcup y}=\bar{x} \sqcap \bar{y}$.

Then (iv) is similar. The last, (v) is from the law of excluded middle and PM4.

Remark 4. If $\forall z(z \bullet x \rightarrow z \bullet y)$ then $x \leqslant y$; if $\forall z(z \bullet x \leftrightarrow z \bullet y)$ then $x=y$.

Why? The same argument for Prop 3(i) shows that if $\forall z(\neg z \bullet y \rightarrow \neg z \bullet x)$, then $\neg \bar{y} \bullet x$, and so $x \leqslant y$ by double negation. Identity follows from the biconditional by PM2. But this does not undermine FACT 2, which claimed a failure of extensionality. There, the claim was that overlapping all the same things is insufficient for identity, and the explanation was that identity requires things have the same non-parts. That is what full complementation and the contraposing biconditional deliver. The proof shows that explicit negation facts are required to confirm identities.

The restricted complement of $y$ in $x$ is

$$
x-y={ }_{\mathrm{df}} x \sqcap \bar{y}
$$

From the existence of fusions, axiom PM5, there is a universal object, $\mathbb{1}$, such that $\forall x(x \leqslant \mathbb{1})$. This object may be thought of as the least upper bound of the $T$ s, where $T$ is a no-place connective that

\footnotetext{
${ }^{24}$ Indeed, that the following fails is instructive: $x \leqslant y$ does not imply that $x \leqslant \bar{y}$. The proof would go by reductio, arguing that if $x \leqslant y \wedge x \leqslant \bar{y}$ then $y \bullet \bar{y}$, which is impossible- but it is not impossible here. And even if it were, we would only have it that $x \leqslant y \vee x \leqslant \bar{y}$, which goes nowhere without disjunctive syllogism. The model in $\S$ Appendix 2 does not satisfy $x \leqslant y \rightarrow x \leqslant \bar{y}$.
} 
is satisfied by everything. Then unrestricted complements can be understood as $\bar{x}=\mathbb{1}-x$. The limiting case $=\mathbb{1}-\mathbb{1}=\mathbb{1} \sqcap \overline{\mathbb{1}}=\overline{\mathbb{1}}$ fails to exist. Were to exist, it would naturally correspond to the least upper bound of the no-place absurdity constant $\perp$, which nothing satisfies on pain of triviality: $\perp \rightarrow A$ for all $A$. There is no least upper bound whatsoever of 'absurd' objects.

A non-paraconsistent algebra for mereotopology has it that $x \sqcap \bar{x}$ always fails to exist. Since the idea of paraconsistency is to allow the possibility of coherent inconsistency, $\mathbb{1} \sqcap \overline{\mathbb{1}}$ fails, but not necessarily for other $x$. We take the notation as invitation for an object satisfying it, and therefore introduce names for a restricted self-complement,

$$
\mathbf{0}_{\mathbf{x}}={ }_{\mathrm{df}} x-x
$$

to keep track of the 'bottom element' for each object $x$ under consideration. For different $a, b, c, \ldots$ there are different $\mathbf{0}_{\mathbf{a}}, \mathbf{0}_{\mathbf{b}}, \mathbf{0}_{\mathbf{c}}, \ldots$ If $a \leqslant x$ then $\mathbf{0}_{\mathbf{a}} \leqslant x$.

In general, $x-x$ is only 'empty' to the extent that $\neg x \bullet \bar{x}$. (Again, by PM4, this always holds, even if $x \bullet \bar{x}$ too.) Unless inconsistent parthood or overlap are trivializing, in the sense of $\perp$, then $\mathbf{0}_{\mathbf{x}}$ can exist. In a small abuse of language, if $x$ has inconsistent overlap, $\mathbf{0}_{\mathbf{x}}$ is non-trivial.

What is $\mathbf{0}_{\mathbf{x}}$ ? It is the distinctive 'shape' of the empty space left when $x$ is gone, the inconsistent residue of $x$ (see $\S 6$ ). The following lemma sheds a bit more light.

Lemma 5. Let $a \leqslant x$. The following hold:

(i) $x-(x-a)=a \sqcup \mathbf{0}_{\mathbf{x}}$

(ii) $x-\mathbf{0}_{\mathbf{x}}=x$

(iii) $\mathbf{0}_{\mathbf{x}}=\mathbf{0}_{\mathbf{x}} \sqcap a$ and $a=a \sqcup \mathbf{0}_{\mathbf{x}}$ if and only if $a=x$

Proof. These are exercises in applying lattice and De Morgan laws, Props 2 and 3. For (i),

$$
\begin{aligned}
x-(x-a) & =x \sqcap \overline{(x \sqcap \bar{a})} \\
& =x \sqcap(\bar{x} \sqcup \overline{\bar{a}}) \\
& =(x \sqcap \bar{x}) \sqcup(x \sqcap a) \\
& =\mathbf{0}_{\mathbf{x}} \sqcup a
\end{aligned}
$$

Then (ii) is a special cases of (i), and (iii) is computed similarly. ${ }^{25}$

A theory of parthood is a good start. Now the parts need to be connected, via topological notions. Relative self-complements will be salient.

\footnotetext{
${ }^{25}$ Hints for calculating: While both $x-a=b$ and $x-b=a$ imply that $x=a \sqcup b$, the converse does not hold, by dint of failure of disjunctive syllogism, which here is $a \sqcap(\bar{a} \sqcup b) \leqslant b$. Indeed, $x=a \sqcup b$ leaves irreducible traces, in that it implies only $x-a=\mathbf{0}_{\mathbf{a}} \sqcup b$ and $x-b=a \sqcup \mathbf{0}_{\mathbf{b}}$. Note that in general cancellation property, $x \sqcap y=x \sqcap z \rightarrow y=z$, is not valid, e.g. $\mathbf{0}_{\mathbf{x}}=\mathbf{0}_{\mathbf{y}}$ does not imply $x=y$.
} 


\section{Topology: Boundaries in Connected Space}

The task of this section is to provide a way to understand how bounded objects can sit in connected space.

5.1. Adding Topological Structure. To make precise the idea of connection, we add unary interior and closure operators that satisfy the Kuratowski closure axioms, ${ }^{26}$

CA1: $x \leqslant c(x)$

CA2: $c(c(x))=c(x)$

CA3: $c(x \sqcup y)=c(x) \sqcup c(y)$

CA4: $c\left(\mathbf{0}_{\mathbf{x}}\right)=\mathbf{0}_{\mathbf{x}}$

when $\mathbf{0}_{\mathbf{x}}$ exists. The dual of $c$ is the interior operator, $i(x)=_{\mathrm{df}} \overline{c(\bar{x})}$. It therefore satisfies $i(x) \leqslant x$, $i(i(x))=i(x), i(x \sqcap y)=i(x) \sqcap i(y)$, and $i(x \sqcup \bar{x})=x \sqcup \bar{x}$. In general, the interior and closure have an orderly relationship,

$$
i(x) \leqslant x \leqslant c(x)
$$

Call $x$ open iff $i(x)=x$ and closed iff $c(x)=x$; then $x$ is both open and closed, or clopen, iff $i(x)=x=c(x)$. By duality of $c$ and $i$, an object will be open iff its complement is closed. ${ }^{27} \mathrm{~A}$ topology on a space is a collection of open objects, closed under fusions and finite products, including the whole space itself, and all the relative self-complements of proper parts of the space.

Lemma 6. Every $\mathbf{0}_{\mathbf{x}}$ in the topology is clopen.

Proof. $\mathbf{0}_{\mathbf{x}}$ is open by the definition of a topology, and closed by CA4: $i\left(\mathbf{0}_{\mathbf{x}}\right)=\mathbf{0}_{\mathbf{x}}=c\left(\mathbf{0}_{\mathbf{x}}\right)$

5.2. Boundaries. Define a boundary operator: for $a \leqslant x$,

$$
\partial(a)=_{\mathrm{df}} c(a) \sqcap c(x-a)
$$

In classical topology, the boundary of the empty space is empty, a space shares its boundary with its complement, and boundaries are fixed points for $\partial$. In the paraconsistent framework, these properties appear as follows.

Proposition 7. Let $x$ be the entire space, and $a \leqslant x$. The following conditions hold:

(i) $\partial(x)=\partial\left(\mathbf{0}_{\mathbf{x}}\right)=\mathbf{0}_{\mathbf{x}}$

(ii) $\partial(\partial(x))=\partial(x)$

(iii) $\partial\left(a \sqcup \mathbf{0}_{\mathbf{x}}\right)=\partial(x-a)$

\footnotetext{
${ }^{26}$ In the classical case, adding CA1-CA4 to HM0-HM6 would result in the mereotopological theory called GEMTC in [Casati and Varzi, 1999, p. 59]. For related structures, see for example, the De Morgan algebras with interior and closure operators as given in [Zhang and Yao, 2007] developed for quite different applications, and based in classical logic.

${ }^{27}$ Additionally, objects may be regular open, meaning that $x=i(c(x))$ or regular closed, meaning that $x=c(i(x))$, though this is not always the case.
} 
Proof. First, note that $\mathbf{0}_{\mathbf{z}} \leqslant z$ for any $z$. For (i), a space shares its boundary with its self-complement by Lemmas 5 and 6 :

$$
\begin{aligned}
\partial\left(\mathbf{0}_{\mathbf{x}}\right) & =c\left(\mathbf{0}_{\mathbf{x}}\right) \sqcap c\left(x-\mathbf{0}_{\mathbf{x}}\right) \\
& =\mathbf{0}_{\mathbf{x}} \sqcap c(x) \\
& =c(x) \sqcap c\left(\mathbf{0}_{\mathbf{x}}\right)=\partial(x)
\end{aligned}
$$

And since $\mathbf{0}_{\mathbf{x}} \leqslant x \leqslant c(x)$, then by transitivity and lattice properties (Prop 2), $\mathbf{0}_{\mathbf{x}} \sqcap c(x)=\mathbf{0}_{\mathbf{x}}$. Then (ii) is obtained by substitution on (i).

These hold only as a special properties for the whole $x$-universe; proper parts $a$ of $x$ are affected by the other parts of $x$, making the general proof fail. But clause (iii) holds for all $a \leqslant x$, and is the raison d'être for relativized bottom elements: since

$$
\begin{aligned}
x-\left(a \sqcup \mathbf{0}_{\mathbf{x}}\right) & =x \sqcap \overline{a \sqcup(x \sqcap \bar{x})} \\
& =x \sqcap(\bar{a} \sqcap(x \sqcup \bar{x})) \\
& =(x \sqcap \bar{a}) \sqcap(x \sqcup \bar{x}) \\
& =x-a
\end{aligned}
$$

then, using Lemma 5 ,

$$
\begin{aligned}
\partial\left(a \sqcup \mathbf{0}_{\mathbf{x}}\right) & =c\left(a \sqcup \mathbf{0}_{\mathbf{x}}\right) \sqcap c\left(x-\left(a \sqcup \mathbf{0}_{\mathbf{x}}\right)\right) \\
& =c(x-(x-a)) \sqcap c(x-a) \\
& =\partial(x-a)
\end{aligned}
$$

Thus, once inconsistency is explicitly tracked, a boundary operator behaves as expected, preserving the intuition that two objects can abut by sharing their boundary.

Boundaries give us an alternative characterization of fundamental notions: the boundary of $a$ is $a$ 's closure without its interior; $a$ with or without its boundary is $a$ 's closure or interior, respectively [Kelley, 1955, p. 46]. Crucially, $a$ is open iff $\partial(a) \leqslant \bar{a}$, and $a$ is closed iff $\partial(a) \leqslant a$. Thus $a$ is both open and closed iff

$$
\partial(a) \leqslant a \sqcap \bar{a}
$$

The idea that clopen objects have empty boundaries has been rather deeply recast. This brings us to our central goal.

5.3. Connectedness. Intuitively, a whole space $x$ is connected iff it cannot be partitioned into non-empty, disjoint, open parts. In mereotopological terms, two objects are connected if they 
overlap, or if one overlaps the closure of the other [Casati and Varzi, 1999, p.52-58]:

$$
x \gtrless y={ }_{\mathrm{df}} x \bullet y \vee x \bullet c(y) \vee c(x) \bullet y
$$

Objects are separated iff they are not connected. More generally, a whole space $x$ is separated iff it has open parts $a, b$ that sum to $x$, but that are not connected;

(1) $x=a \sqcup b$

(2) $\neg a æ b$

(3) $a$ and $b$ are both open

A space is self-connected iff it is not separated:

$$
§(x)=\mathrm{df}_{\mathrm{df}} \forall a \forall b(x \neq a \sqcup b \vee a \preccurlyeq b \vee \partial(a) \$ x-a \vee \partial(b) \$ x-b)
$$

Self-connected spaces exist: paradigmatically, $\mathbb{R}$ is the real number line with standard features, as paraconsistently developed in [McKubre-Jordens and Weber, 2012].

The sketch up to here suggests that paraconsistent mereotopology is robust enough to sustain a mathematical conception of a (self) connected space. Our main theorem now shows that the concept of connected space has been relaxed enough to allow closed objects in contact with one another.

Theorem 8. Some connected spaces have proper parts that are both open and closed.

Proof. Figure 1 ('A Simple Universe') is the paradigm case. The following more pathological example is to prove the point.

For some $a$ for which $a \bullet \bar{a}$, take our space to be a single point, the object $\mathbf{0}_{\mathbf{a}}$. This is a connected space, since $\mathbf{0}_{\mathbf{a}} \Varangle \mathbf{0}_{\mathbf{a}}$. By Lemma $6, \mathbf{0}_{\mathbf{a}}$ is open and closed. But since $\mathbf{0}_{\mathbf{a}} \bullet \mathbf{0}_{\mathbf{a}}$ (since everything overlaps itself by PM1), and $\neg \mathbf{0}_{\mathbf{a}} \bullet \mathbf{0}_{\mathbf{a}}$ (since nothing overlaps $\mathbf{0}_{\mathbf{a}}$ by Prop 3 ), then

$$
\mathbf{0}_{\mathbf{a}} \leqslant \mathbf{0}_{\mathbf{a}} \wedge \exists z\left(z \bullet \mathbf{0}_{\mathbf{a}} \wedge z 1 \backslash \mathbf{0}_{\mathbf{a}}\right)
$$

showing that $\mathbf{0}_{\mathbf{a}}$ is a proper part of the space, as required.

Since the three condition for being separated also hold $-\mathbf{0}_{\mathbf{a}}=\mathbf{0}_{\mathbf{a}} \sqcup \mathbf{0}_{\mathbf{a}}, \neg \mathbf{0}_{\mathbf{a}} \gtrless \mathbf{0}_{\mathbf{a}}$, and $\mathbf{0}_{\mathbf{a}}$ is open - then we have our last fact, an inconsistency at the level of theorizing about inconsistent boundaries:

Corollary 9. There are connected spaces upon which exists a partition into disjoint, non-empty open parts. Some spaces are both connected and not.

5.4. Peirce's Puzzle Revisited. There are empty parts, indexed to a space, such that boundaries of clopen objects are both empty and not, and the whole space ends up being both connected and not. This existence claim suffices to show that on at least some spaces, the naive intuitions about objects, boundaries, and connection can all be retained. 
These results amount to a 'proof of concept'. To illustrate how it helps with our initial problem, return to the Great Red Spot on Jupiter. The mathematical modeling tools at our disposal now allow the Spot to have an inconsistent boundary. This means it is possible to have all the intuitive constraints hold jointly, as follows. The Spot and its complement share a boundary, modulo inconsistency of its relative self-complement (Proposition 7). Any points on the boundary are included in the Spot, and so are red; boundary points are also included in the Spot's complement, and so are not red. Nevertheless, the whole surface of Jupiter can remain connected (Theorem 8). Inconsistent boundaries are enough to preserve SYMMETRY $(\S 2.1)$, without separating their host space $(\S 2.2)$.

To conclude, then, it remains to turn to a few questions about the meaning and role of empty parts in this framework.

\section{Conclusion: Empty Parts}

By accepting empty parts in a suitable form, namely as the inconsistent aspect of an object, enough technical control is restored in the algebra to make some sense of puzzles in the metaphysics of naive space. Paraconsistent mereotopology puts focus on highly localized properties: for an object and its complement to share a boundary completely, we must consider the empty parts of that object explicitly. Then, symmetry is restored and connected spaces can have closed, nonempty subspaces. The question is, what to make of this notion of emptiness?

The usual point-set definition of a topology makes indispensable use of the empty set. To prevent confusion, we recall the difference between an empty set and an empty object: $\varnothing$ is still something, namely, a set. This is no more mysterious than the number zero. (The quantity of pink elephants exists, not in spite of but because of the fact that no pink elephants exist.) Matters are a little less clear with an empty object, which is nothing. Even that locution sounds incoherent: 'it' overlaps everything, 'it' is a part of everything, but only because 'it' is the punchline of a Lewis Carroll joke. 'It' isn't. Zero and the empty set might represent null things, but a null thing is neither a number nor a set. ${ }^{28}$

Nevertheless, an empty object seems almost forced on us, by the algebraic structure that a decent topology demands. Many versions of mereology have a universe, an individual of which everything is a part, mainly because it improves the algebraic structure; in fact, the use of an empty object is not so uncommon. ${ }^{29}$ While algebraic utility is presented as if it were the crudest of reasons for supposing a null individual to exist, the same was once said of zero, negative integers, and complex numbers. The argument from algebraic utility may be enough on its own, to justify the involvement of empty parts. But we think that in the paraconsistent setting, there is a more direct explanation.

\footnotetext{
${ }^{28} \mathrm{~A}$ null individual is proposed by Carnap as the designatum of an empty name, where he says that the null thing "corresponds to the null class of space-time points" [Carnap, 1947, p. 36].

29 [Casati and Varzi, 1999, p. 45]. Arntzenius uses a bottom element with only parenthetical comment [Arntzenius, 2012, p. 136]. Roeper [Roeper, 1997, p. 253] invokes one in order to use the Stone Representation Thereom. Forrest [Forrest, 2002, p. 81] utilizes a null object, which he dismisses as 'fictitious'.
} 
We can begin to make conceptual, and not merely instrumental, sense of what these objects are like, tying together these two mereological and topological threads as follows.

In apparent contrast to empty parts, the notion of a boundary is much more accepted by mereologists $(\S 3)$. But, we have seen, a boundary in connected space is best thought of as involving the overlap of something with its complement. The collusion of SYMMETRY and SEPARATION suggest that boundaries are inconsistent objects. This was our puzzle. On classical-leaning views, of course, inconsistent objects are a nonsense. There simply are no such things, not even as fictions; inconsistent theories have no models. And so, on these views, boundaries remain problematic. Not so on a glutty, paraconsistent view: there are inconsistent objects - or at least, objects that faithfully track real, inconsistent properties. But bear in mind that $\neg(A \wedge \neg A)$ is still a theorem of paraconsistent logic; so formally, the objects corresponding to inconsistency are (both) empty (and not). Even dialetheically, inconsistency is "nowhere and nowhen." ${ }^{30}$ The explanation, then, for the presence of empty objects in paraconsistent mereotopology is that inconsistency turns up in the mathematics as emptiness. Boundaries must be empty; they cannot be empty. The apparent, and perhaps strange, appeal to empty parts is in fact an acknowledgement of the contradiction inherent in boundaries.

Mereotopologically, then, empty parts come to the fore around boundaries in connected space that is, when they aren't so empty after all. When it exists, the bottom $\mathbf{0}_{\mathbf{a}}$ is emphatically not going to be a part of every other thing; the alternative would validate the inference of ex contradictione quodlibet. A main tenet of paraconsistency is that not all falsity is absurd; and so some objects can be paraconsistently empty (to the degree 'false') without being purely empty (to the degree ' $\perp$ '). Classical mereotopologies cannot make sense of an empty part because they are only looking at the most bizarre, singularly absurd one (namely, ), of which we agree there is little sense to be made. For a non-classical mereotopology, meanwhile, there are lots of empty parts around, many of which are day-to-day called boundaries.

Acknowledgements: Thanks to audiences at the 25 Years In Contradiction Conference at Glasgow University, the Metaphysical Indeterminacy Worskshop VI at the University of Leeds, the 2009 Mereology Workshop at the University of St Andrews, the Australian Metaphysics Conference at Kioloa, the Pukeko Logic Group, and the University of Otago for comments and discussion on various versions of these ideas. Thanks especially to Ralf Bader, Guillermo Badia, Elizabeth Barnes, Can Baskent, Jc Beall, Franz Berto, Ben Burgis, Ross Cameron, Filippo Casati, Katherine Hawley, Cory Johnson, Manuel Lechthaler, Hitoshi Omori, Graham Priest, Adam Reiger, David Ripley, Marcus Rossberg, Lionel Shapiro, Stewart Shapiro, Reed Solomon, Diego Tajer, Jason Turner, Gabriel Uzquiano, Achille Varzi, Alan Weir, Robbie Williams, and Gareth Young. Research on this paper was supported by a grant from the Marsden Fund, Royal Society of New Zealand.

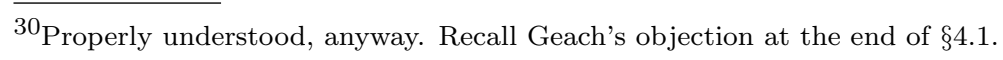




\section{Appendix 1: LogiC}

The logic for our paraconsistent mereology is the weak relevant logic DKQ, previously applied to paraconsistent set theory; see [Priest, 2006, p.251], [Weber, 2012]. Set theory in DKQ is nontrivial - it has a relative 'consistency' proof - because it has a classical model in which some sentence is not satisfied [Brady, 2006].

The language is that of first order logic with identity and membership. The usual shorthand is used: $A \vee B$ for $\neg(\neg A \wedge \neg B) ; A \leftrightarrow B$ for $(A \rightarrow B) \wedge(B \rightarrow A) ; \exists$ is $\neg \forall \neg$.

Axioms. All instances of the following schemata are theorems:

$$
\begin{array}{lll}
I & A \rightarrow A & \\
\text { IIa } & A \wedge B \rightarrow A & \\
\text { IIb } & A \wedge B \rightarrow B & \\
I I I & A \wedge(B \vee C) \rightarrow(A \wedge B) \vee(A \wedge C) & \text { (distribution) } \\
I V & (A \rightarrow B) \wedge(B \rightarrow C) \rightarrow(A \rightarrow C) & \text { (conjunctive syllogism) } \\
V & (A \rightarrow B) \wedge(A \rightarrow C) \rightarrow(A \rightarrow B \wedge C) & \\
V I & (A \rightarrow B) \leftrightarrow(\neg B \rightarrow \neg A) & \text { (contraposition) } \\
V I I & A \leftrightarrow \neg \neg A & \text { (double negation elimination) } \\
V I I I & A \vee \neg A & \text { (excluded middle) } \\
I X & (\forall x) A \rightarrow A(a / x) & \\
X & (\forall x)(A \rightarrow B) \rightarrow(A \rightarrow(\forall x) B) & \text { (with } x \text { not free in } A \text { ) } \\
X I & (\forall x)(A \vee B) \rightarrow A \vee(\forall x) B & \text { (with } x \text { not free in } A \text { ) }
\end{array}
$$

The law of excluded middle implies the law of non-contradiction, $\neg(A \wedge \neg A)$.

Rules. The following rules are valid:

$$
\begin{array}{lll}
I & A, B \vdash A \wedge B & \text { (adjunction) } \\
\text { II } & A, A \rightarrow B \vdash B & \text { (modus ponens) } \\
\text { III } & A \vdash(\forall x) A & \text { (universal generalization) } \\
I V & A \rightarrow B, C \rightarrow D \vdash(B \rightarrow C) \rightarrow(A \rightarrow D) & \text { (hypothetical syllogism) } \\
V & x=y \vdash A(x) \leftrightarrow A(y) & \text { (substitution) }
\end{array}
$$

Structural Rules. The following meta-rules preserve validity:

$$
\frac{A \vdash B}{A \vee C \vdash B \vee C} \quad \frac{A \vdash B}{\exists x A \vdash \exists x B}
$$

This validates argument by cases, and, excluded middle, reductio:

$$
\frac{A \vdash C \quad B \vdash C}{A \vee B \vdash C} \quad \frac{A \vdash \neg A}{\vdash \neg A}
$$


Enthymematic conditional. Add a $t$-constant that obeys the two-way rule

$$
A \dashv t \rightarrow A
$$

The $t$-constant may be thought of as the conjunction of all truths. Then define $A \mapsto B:=A \wedge t \rightarrow B$. This defined operator has the property of weakening:

$$
A \vdash B \mapsto A
$$

However, it does not contrapose: $A \mapsto B \nvdash \neg B \mapsto \neg A$.

Inconsistency and absurdity. For ease of presentation, we adopt an axiom asserting the truth of at least one contradiction:

$$
p \wedge \neg p
$$

for some fixed $p$. After all, the axiom set PM is no more than a classical set re-written with paraconsistent conditionals. So there is no reason to expect that it generates a contradiction on its own. Within the language of PM so defined, then, there is at least one $\mathbf{0}_{\mathbf{x}}$, which is required for the theorems above. Otherwise they would simply read that if $\mathbf{0}_{\mathbf{x}}$ exists then..., which is classically true too, by ex falso quodlibet! By the same token, to ensure that there is at least one unacceptable sentence, a constant $\perp$ is added to the language, and a final axiom

$$
\perp \rightarrow A
$$

asserts that $\perp$ is never designated.

\section{ApPendix 2: Non-Triviality}

Here we present an interpretation $\mathcal{M}$ of the formal theory, showing that even if it is inconsistent it is not trivial. The presentation and proof that $\mathcal{M}$ is a non-trivial model are conducted in standard classical logic, following standard practice in the relevant logic and mathematics literature (e.g. [Priest, 2006, Ch 17]); the model is suggested by Meyer and Mortensen's two-element model for relevant arithmetic. This model is not presented as the intended model of paraconsistent mereology. The axiomatization PM is not complete with respect to the model. Our only concern is soundness, which in the present context is non-triviality.

Fact. Paraconsistent mereology has a model.

Proof. To interpret the logic, we use truth tables for the paraconsistent logic RM3:

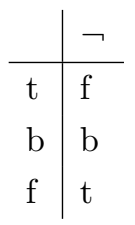

\begin{tabular}{c|ccc}
$\wedge$ & $t$ & $b$ & $f$ \\
\hline$t$ & $t$ & $b$ & $f$ \\
$b$ & $b$ & $b$ & $f$ \\
$f$ & $f$ & $f$ & $f$
\end{tabular}

\begin{tabular}{l|lll}
$\rightarrow$ & $t$ & $b$ & $f$ \\
\hline$t$ & $t$ & $f$ & $f$ \\
$b$ & $t$ & $b$ & $f$ \\
$f$ & $t$ & $t$ & $t$
\end{tabular}


The designated values of RM3 are t and b. RM3 validity is preservation of designated values. An argument is invalid if there is a valuation making all the premises designated, but not the conclusion. The conditional of DK does not have a truth table, but DK is sound with respect to RM3. Anything invalid by RM3 truth tables is DK invalid.

The enthymematic conditional, $A \mapsto B$, should be thought of as having an antecedent that is no more than ' $\mathrm{b}$ ', since the connective $t$ is the conjunction of all truths, and some of these are false [Beall et al., 2011]. So the conditional never has an antecedent that is $t$ only, and the only way it can get an $f$ is if the consequent is $f$.

To define a model $\mathcal{M}=\langle D, V\rangle$, let the domain $D=\{0,1\}$ and $V(\leqslant)$ be as follows:

\begin{tabular}{c|cc}
$\leqslant$ & 0 & 1 \\
\hline 0 & $\mathrm{~b}$ & $\mathrm{t}$ \\
1 & $\mathrm{f}$ & $\mathrm{b}$
\end{tabular}

That is, $1 \leqslant 0$ is just false, $0 \leqslant 1$ is just true, and it is both true and false that things are parts of themselves. For identity,

\begin{tabular}{c|cc}
$=$ & 0 & 1 \\
\hline 0 & $\mathrm{~b}$ & $\mathrm{f}$ \\
1 & $\mathrm{f}$ & $\mathrm{b}$
\end{tabular}

Because the domain is finite, quantifiers $\forall$ and $\exists$ can be understood as conjunctions and disjunctions, respectively.

Now it is just a matter of verifying the axioms, showing that they have designated values.

For PM1, $V(0 \leqslant 0)=V(1 \leqslant 1)=b$, so $V(0 \leqslant 0 \wedge 1 \leqslant 1)=b$ and therefore $V(\forall x(x \leqslant x))=b$.

For PM2, anti-symmetry, we consider all the possible values of $x$ and $y$ :

$$
\begin{aligned}
& V(0 \leqslant 1 \wedge 1 \leqslant 0)=V(0=1)=f \\
& \text { or } \quad V(0 \leqslant 0 \wedge 0 \leqslant 0)=V(0=0)=b \\
& \text { or } \quad V(1 \leqslant 1 \wedge 1 \leqslant 1)=V(1=1)=b
\end{aligned}
$$

In all cases, the required biconditional holds.

For PM3, transitivity, the only case where the consequent fails is $V(1 \leqslant 0)=f$. Then the antecedent $V(1 \leqslant y \wedge y \leqslant 0)=f$ whether $y$ is 1 or 0 . There is no case where $V(x \leqslant y \wedge y \leqslant x)=t$.

For PM4, we set 1 as the complement of 0 , and vice versa. Well, $V\left(\begin{array}{lll}1 & 1 & 0\end{array}\right)=b$ because

$$
V(0 \$ 0 \vee 0 \$ 1)=b
$$

and

$$
V(1 \$ 0 \vee 1 \$ 1)=t
$$


so the conjunction of the two is $b$. And to show $\forall y((\neg y \bullet 0 \rightarrow y \leqslant 1) \wedge(\neg y \bullet 1 \rightarrow y \leqslant 0))$, we have

$$
\begin{aligned}
& V(0 \not h 0 \rightarrow 0 \leqslant 1)=t \\
& V(01 \downarrow 1 \rightarrow 0 \leqslant 0)=b \\
& V(1 \not h 0 \rightarrow 1 \leqslant 1)=b \\
& V(111 \rightarrow 1 \leqslant 0)=t
\end{aligned}
$$

making the conjunction of these four $\mathrm{b}$, designated. To show the converses of all these, note that they all work as $\rightarrow$ except the first, which would be $V(0 \leqslant 1 \rightarrow 0 \Uparrow k)=f$ since the antecedent is $\mathrm{t}$ and the consequent is $\mathrm{b}$. But the $\mapsto$ rendering drops the antecedent down to $\mathrm{b}$ and the sentence is satisfied. The same reasoning applies to show that 0 is the complement of 1 .

For PM5, we need a witness to play lub. Now, $V(A)$ is t, b, or $\mathrm{f}$. Then take the lub of the $A \mathrm{~s}$ to be 0 when $A$ is $f$, and the lub of the $A$ s is 1 otherwise. In more detail, consider the case where $V(A)$ is $\mathrm{t}$ or b. Well, $V(x \leqslant 1)$ is always designated, so $V(A \mapsto x \leqslant 1)$ is always designated. And then 1 is least since a check of truth tables shows

$$
\begin{aligned}
& V((A \mapsto 0 \leqslant 1) \wedge(A \mapsto 1 \leqslant 1) \mapsto 1 \leqslant 1)=b \\
& V((A \mapsto 1 \leqslant 0) \wedge(A \mapsto 0 \leqslant 0) \mapsto 1 \leqslant 0)=t
\end{aligned}
$$

Otherwise, $V(A)=f$. Then $V(A \mapsto 0 \leqslant 0)=V(A \mapsto 1 \leqslant 0)=t$. And to show that 0 is least here,

$$
\begin{aligned}
& V((A \mapsto 0 \leqslant 0) \wedge(A \mapsto 1 \leqslant 0) \mapsto 0 \leqslant 0)=t \\
& V((A \mapsto 0 \leqslant 1) \wedge(A \mapsto 1 \leqslant 1) \mapsto 0 \leqslant 1)=t
\end{aligned}
$$

Thus PM5 is always designated.

For PM6, $V(1 \$ 1)=b$ and $V(0 \$ 0)=b$, so $V(\exists x(x \$ 1))$ and $V(\exists x(x \$ 0))$ are both designated; and then so is their conjunction.

For PM7, we have $V(0 \bullet 0)=V(0 \bullet 1)=V(1 \bullet 0)=b$, and $V(1 \bullet 1)=t$. Then the cases are all designated:

$$
\begin{aligned}
& 0 \leqslant 0 \mapsto(0 \bullet 0 \rightarrow 0 \bullet 0) \wedge(1 \bullet 0 \rightarrow 1 \bullet 0) \\
& 0 \leqslant 1 \mapsto(0 \bullet 0 \rightarrow 0 \bullet 1) \wedge(1 \bullet 0 \rightarrow 1 \bullet 1) \\
& 1 \leqslant 0 \mapsto(0 \bullet 1 \rightarrow 0 \bullet 0) \wedge(1 \bullet 1 \rightarrow 1 \bullet 0) \\
& 1 \leqslant 1 \mapsto(0 \bullet 1 \rightarrow 0 \bullet 1) \wedge(1 \bullet 1 \rightarrow 1 \bullet 1)
\end{aligned}
$$

noting that the second case would not be designated with an $\rightarrow$ as the main connective.

For the topology, we need to interpret Kuratowski's closure axioms. This can be done very easily: let

$$
V(c(0)=0)=V(c(1)=1)=t
$$


Again, this overdoes it, but suffices to show the existence of a model. For CA1, this follows from PM1; for CA2, $c(c(x))=c(x)$ for both 0 and 1 ; and so forth. The interior operator, $i(x)$, is then $\overline{c(\bar{x}})=\overline{\bar{x}}=x$, which satisfiers the dual interior properties.

Fact. Not every sentence of the language of PM is provable.

Proof. The sentence $\forall x \forall y(x \leqslant y)$ gets value $\mathrm{f}$ in the model. All we need is one; but there are many, e.g. $\forall x \forall y(x \bullet y \rightarrow x \leqslant y)$ gets $\mathrm{f}$, too.

This completes the proof.

\section{REFERENCES}

[Arntzenius, 2012] Arntzenius, F. (2012). Space, Time, and Stuff. Oxford University Press.

[Beall et al., 2011] Beall, J., Priest, G., and Weber, Z. (2011). Can u do that? Analysis, 71(2):280 - 285.

[Bolzano, 1950] Bolzano, B. (1950). Paradoxes of the Infinite. Routledge, London.

[Brady, 2006] Brady, R. (2006). Universal Logic. CSLI Publications, Stanford.

[Brentano, 1988] Brentano, F. (1988). Philosophical Investigations on Space, Time and the Continuum. Croom Helm, London.

[Bunge, 1966] Bunge, M. (1966). On null individuals. Journal of Philosophy, 63:776-778.

[Bunt, 1985] Bunt, H. C. (1985). Mass Terms and Model-Theoretic Semantics. Cambridge University Press, Cambridge.

[Carnap, 1947] Carnap, R. (1947). Meaning and Necessity. University of Chicago Press.

[Casati and Varzi, 1999] Casati, R. and Varzi, A. C. (1999). Parts and Places: The Structures of Spatial Representation. MIT Press, Cambridge (MA).

[Chisholm, 1984] Chisholm, R. M. (1984). Boundaries as dependent particulars. Grazer philosophische Studien, 10:87-95.

[Clarke, 1985] Clarke, B. L. (1985). Individuals and points. Notre Dame Journal of Formal Logic, 26:61-75.

[Dainton, 2010] Dainton, B. (2010). Time and Space. McGill-Queen's University Press. Second edition.

[Dummett, 2000] Dummett, M. (2000). Is time a continuum of instants? Philosophy, 75(4):497 - 515.

[Forrest, 2002] Forrest, P. (2002). Non-classical mereology and its application to sets. Notre Dame Journal of Formal Logic, 43:79-94.

[Geach, 1949] Geach, P. (1949). On rigour in semantics. Mind, 58(232):518-522.

[Hestevold, 1986] Hestevold, H. S. (1986). Boundaries, surfaces, and continuous wholes. Southern Journal of Philosophy, 24:235-245.

[Hocking and Young, 1961] Hocking, J. G. and Young, G. S. (1961). Topology. Dover.

[Hovda, 2009] Hovda, P. (2009). What is classical mereology? Journal of Philosophical Logic, 38(1):55-82.

[Hudson, 2005] Hudson, H. (2005). The Metaphysics of Hyperspace. Oxford University Press.

[Kelley, 1955] Kelley, J. L. (1955). General Topology. Springer-Verlag, New York.

[Koslicki, 2008] Koslicki, K. (2008). The Structure of Objects. Oxford University Press.

[Martin, 1965] Martin, R. (1965). Of time and the null individual. Journal of Philosophy, 62:723-736.

[McKubre-Jordens and Weber, 2012] McKubre-Jordens, M. and Weber, Z. (2012). Real analysis in paraconsistent logic. Journal of Philosophical Logic, $\mathrm{x}(\mathrm{x}): \mathrm{xx}$.

[Oliver and Smiley, 2013] Oliver, A. and Smiley, T. (2013). Zilch. Analysis, 73:601-613.

[Peirce, 1933] Peirce, C. (1933). The logic of quantity. In Hartshorne, C. and Weiss, P., editors, Collected Papers of Charles Sanders Peirce, volume IV. Harvard University Press, Cambridge, MA. 
[Pratt-Harmon, 2007] Pratt-Harmon, I. (2007). First order mereotopology. In Aiello, M., Pratt-Harmon, I. E., and van Benthem, J., editors, Handbook of Spatial Logics, pages 13-98. Springer, Dordrecht.

[Priest, 2006] Priest, G. (2006). In Contradiction. Oxford University Press., 2nd edition.

[Priest, 2014] Priest, G. (2014). Much ado about nothing. Australasian Journal of Logic, 11(2):146-158.

[Putnam, 1994] Putnam, H. (1994). Peirce's continuum. In Ketner, K., editor, Peirce and Contemporary Thought:

Philosophical Inquiries. Fordham University Press.

[Randell et al., 1992] Randell, D., Cui, Z., and Cohn, A. (1992). A spatial logic based on regions and connection. In B. Nebel, e. a., editor, Principles of Knowledge Representation and Reasoning: Proceedings of the Third International Conference, pages 165-176, Cambridge, MA.

[Roeper, 1997] Roeper, P. (1997). Region-based topology. Journal of Philosophical Logic, 26:251-309.

[Rogers, 1995] Rogers, J. (1995). The Giant Planet Jupiter. Cambridge University Press.

[Sider, 2000] Sider, T. (2000). Simply possible. Philosophy and Phenomenological Research, 60(3):585—590.

[Simons, 1987] Simons, P. M. (1987). Parts: A Study In Ontology. Clarendon, Oxford.

[Smith, 1997] Smith, B. (1997). Boundaries: An essay in mereotopology. In Hahn, L., editor, The Philosophy of Roderick Chisholm, Library of Living Philosophers, pages 534-561. Open Court, LaSalle.

[Sorensen, 1986] Sorensen, R. A. (1986). Transitions. Philosophical Studies, 50:187-193.

[Stell and Worboys, 1997] Stell, J. and Worboys, M. (1997). The algebraic structure of sets of regions. In Spatial Information Theory: A Theoretical Basis for GIS, volume 1329, pages 163-174. Springer, Berlin.

[Varzi, 1997] Varzi, A. (1997). Boundaries, continuity, and contact. Nô̂s, 31:26 - 58.

[Varzi, 2004] Varzi, A. (Spring 2004). Boundary. In Zalta, E. N., editor, The Stanford Encyclopedia of Philosophy.

[Weber, 2012] Weber, Z. (2012). Transfinite cardinals in paraconsistent set theory. Review of Symbolic Logic, $5(2): 269-293$.

[Whitehead, 1919] Whitehead, A. N. (1919). An Enquiry Concerning the Principles of Human Knowledge. Cambridge University Press, Cambridge.

[Zhang and Yao, 2007] Zhang, X. and Yao, G. (2007). Generalized rough set model on de morgan algebras. volume 0, page 205, Los Alamitos, CA, USA. IEEE Computer Society.

[Zimmerman, 1996] Zimmerman, D. (1996). Could extended objects be made out of simple parts? An argument for atomless gunk. Philosophy and Phenomenological Research, 56:1-29. 